\title{
多源不确定性下平面变胞机构运动可靠性分析
}

\author{
刘胜利 $^{1}$ 王兴东 $^{1}$ 孔建益 $^{1}$ 曾 $^{\text {婷 }^{2}}$ 唐 伟 $^{1}$ \\ (1. 武汉科技大学冶金装备及其控制教育部重点实验室 武汉 430081; \\ 2. 中国航天科技集团公司北京卫星制造厂 北京 100080)
}

\begin{abstract}
摘要: 变胞机构可根据环境和工况的变化及需求, 进行重组和重构, 在机器人领域得到广泛应用。变胞机构可变拓扑结构特 性, 使得分析多源不确定性下变胞机构全构态运动可靠性更为困难。以多源不确定性下平面五杆变胞机构为研究对象, 提出 一种适应交变温度工况的变胞机构全构态运动可靠性分析方法。建立温度载荷下连杆制造公差-热变形、关节配合公差-热变形 的耦合模型，采用服从截断正态分布的随机参数和区间参数描述连杆尺寸、转动副和移动副间隙的不确定性，基于变胞特性 建立变胞机构全构态运动误差传递模型, 结合区间优化算法和蒙特卡洛算法建立多源不确定性下变胞机构全构态运动可靠性 模型。基于提出的方法, 分析了交变温度工况下变胞机构全构态运动可靠性分布规律, 并分析了不同孔轴配合、连杆公差等级 及其热膨胀系数对变胞机构全构态运动可靠性的影响规律。
\end{abstract}

关键词: 平面变胞机构; 多源不确定性; 交变温度; 运动可靠性

中图分类号: TH122

\section{Kinematic Reliability Analysis of Planar Metamorphic Mechanism with Multi-source Uncertainties}

\author{
LIU Shengli $^{1} \quad$ WANG Xingdong $^{1} \quad$ KONG Jianyi $^{1} \quad$ ZENG Ting $^{2} \quad$ TANG Wei $^{1}$ \\ (1. Key Laboratory of Metallurgical Equipment and Control Technology, Wuhan University of Science and \\ Technology, Wuhan 430081; \\ 2. Beijing Spacecrafts, China Aerospace Science and Technology Corporation, Beijing 100080)
}

\begin{abstract}
Metamorphic mechanism can reorganize and reconstruct itself according to the changes and needs of environment and working conditions, and it has been widely used in the field of robot. The variable topological structure of metamorphic mechanism makes it more difficult to analyze the reliability of full configuration motion of metamorphic mechanism under multi-source uncertainty. A method for reliability analysis of planar five-bar metamorphic mechanism under multiple source uncertainties is presented. The coupling model of link manufacturing tolerance, joint tolerance and thermal deformation is set up under the temperature load, adopting obey truncated normal distribution random parameters and interval parameters to describe the length of link, the uncertainty of the clearance of revolute and prismatic, combined with the metamorphic characteristics to establish full-configuration motion error transfer model, based on interval method and Monte Carlo method, establishing full-configuration motion reliability model with multi-source uncertainties. Based on the proposed method, the kinematic reliability distribution law of the configuration-complete and sub-configuration of the metamorphic mechanism under alternating temperature conditions was analyzed, and the influence law of different joint hole shaft fit, connecting rod tolerance grade and thermal expansion coefficient on the kinematic reliability of the metamorphic mechanism is analyzed.
\end{abstract}

Key words: planar metamorphic mechanism; multi-source uncertainties; alternating temperature; kinematic reliability

\section{0 前言}

变胞机构的可变拓扑结构特性使其能够根据环

\footnotetext{
* 国家自然科学基金(51875418)、国家自然科学基金联合基金(U1737207) 和武汉科技大学国防预研基金(GF201906)资助项目。20210110 收到初 稿, 20210520 收到修改稿
}

境和工况的变化以及任务需求, 进行自我重组和重 构, 在航空航天、机器人和制造业等领域具有广阔 的应用前景 ${ }^{[1-2]}$ 。运动变量误差、制造和装配误差、 关节间隙和热变形等随机或认知不确定因素 ${ }^{[3-5]}$, 均 会导致机构的真实运动偏离期望运动, 降低机构的 运动可靠性。运动可靠性是评价机构运动精度的重 
要性能指标, 分析不确定性下变胞机构的运动可靠 性可有效指导机构的设计。

金国光等 ${ }^{[6]}$ 基于矩阵法建立了变胞机构运动 学模型, 对变胞机构进行了构态分析。ZHANG 等 ${ }^{[7]}$ 基于多学科优化设计方法, 以结构误差最小 为目标对平面五杆变胞机构进行尺寸综合。

ZHANG 等 ${ }^{[8]}$ 建立了并联变胞机构运动学分析的 数学模型, 对不同构型的变胞机构进行正逆运动 学分析。HU 等 ${ }^{[9]}$ 基于螺旋理论建立了空间曲面火 焰切割变胞机构的运动误差模型。张满慧等 ${ }^{[10]}$ 考 虑结构误差、运动变量误差及运动副间隙的综合 影响, 建立空间变胞机构的全构态运动误差模型。 国内外对变胞机构的研究较少考虑不确定性因素 的影响, 并结合变胞机构变拓扑结构特性对其进 行运动学分析。

张义民等 ${ }^{[11]}$ 提出了一种不完全概率信息的平 面连杆机构运动精度稳健性设计方法。陈放等 ${ }^{[12]}$ 基于联合概率方法提出了平面轨迹机构时变可靠 性分析方法。PANDEY 等 ${ }^{[13]}$ 用最大熵原理推导机 器人位置误差的极值分布, 分析了机器人的系统 可靠度。ZHAN 等 ${ }^{[14-15]}$ 对混合不确定下平面并 联机构进行运动误差及其可靠性分析。ZHANG 等 ${ }^{[16-17]}$ 基于首次穿越法提出了函数生成机构的时 变可靠性分析方法, 并进一步通过包络法分析 了含关节间隙函数生成机构时变可靠性。WANG 等 ${ }^{[18]}$ 基于包络函数和 Vine-Couple 函数进行了机 构时变可靠性分析。GENG 等 ${ }^{[19]}$ 基于非概率区间 过程模型和首次穿越理论, 提出了一种时变可靠 性评估方法。国内外针对不确定性下机构运动可 靠性研究主要集中在四杆机构和串、并联机构等 机构上, 而对变胞机构运动可靠性研究关注较少。 不同于串、并联机构等单构态机构, 变胞机构会 在不同拓扑结构间进行切换, 需结合变胞特性建 立全构态运动可靠性模型。

本文综合考虑关节间隙、尺寸公差、关节和连 杆的热变形等多源不确定性, 采用随机参数和区间 参数表征不确定性影响因素, 结合变胞特性建立平 面五杆变胞机构全构态运动误差传递模型, 基于区 间优化算法和蒙特卡罗模拟方法(Monte Carlo simulation, MCS) 建立混合不确定性下变胞机构全 构态运动可靠性模型。以平面五杆变胞机构为例, 分析交变温度工况下计及多源不确定性的变胞机构 全构态运动可靠性, 并从设计和制造的角度分析孔
轴配合、连杆公差等级及其热膨胀系数对变胞机构 全构态运动可靠性的影响规律。

\section{1 平面五杆变胞机构构态描述}

平面五杆变胞机构简图见图 $1 \mathrm{a} 、 1 \mathrm{~b}$ ，该机构能 改变拓扑结构, 实现曲柄滑块机构和四连杆机构相 互转换。变胞机构构态变化示意图见图 1c, 当变胞 关节 $C$ 锁定且滑块解锁, 视作构态 1(曲柄滑块机 构); 当滑块接触到挡板, 变胞关节 $C$ 解锁且滑块锁 定, 变胞机构由构态 1 切换为构态 2 (四连杆机构); 当变胞关节 $C$ 回到初始夹角, 此时变胞关节 $C$ 锁定 且滑块解锁, 变胞机构由构态 2 切换为构态 1 。曲 柄每周转一次, 会完成两次构态切换, 构成一个工 作周期。

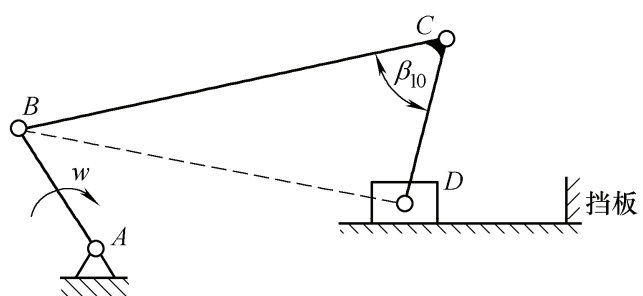

(a) 构态 1 : 曲柄滑块机构

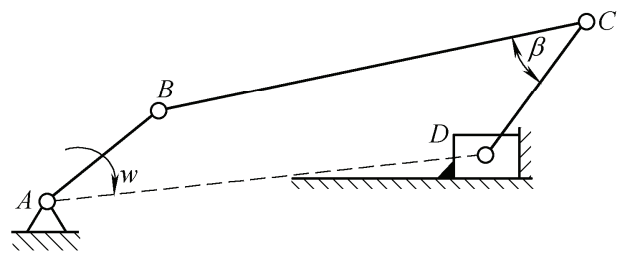

(b) 构态 2 : 四连杆机构

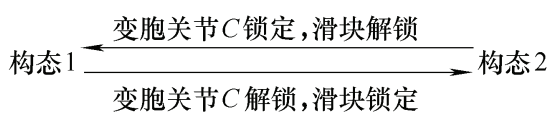

(c) 构态变化示意图

图 1 平面五杆变胞机构机构简图及构态变化示意图

\section{2 不确定性影响因素建模}

本文综合考虑尺寸公差、关节间隙、关节和连 杆的热变形等多源不确定性影响因素, 并分别对其 进行不确定性数学建模。含多源不确定性平面五杆 变胞机构位姿模型如图 2 所示。其中, $L_{1} 、 L_{2} 、 L_{3} 、 L_{5}$ 为连杆尺寸, $H$ 为偏心距, $w 、 z$ 为滑块宽和高, $h$ 为导轨宽, $W$ 为铰点 $A$ 到挡板间距离, $\theta_{1}$ 为曲柄转 角, $\beta_{10}$ 为变胞关节 $C$ 初始夹角, 杆 $G F$ 上点 $F$ 为 变胞机构输出点。 


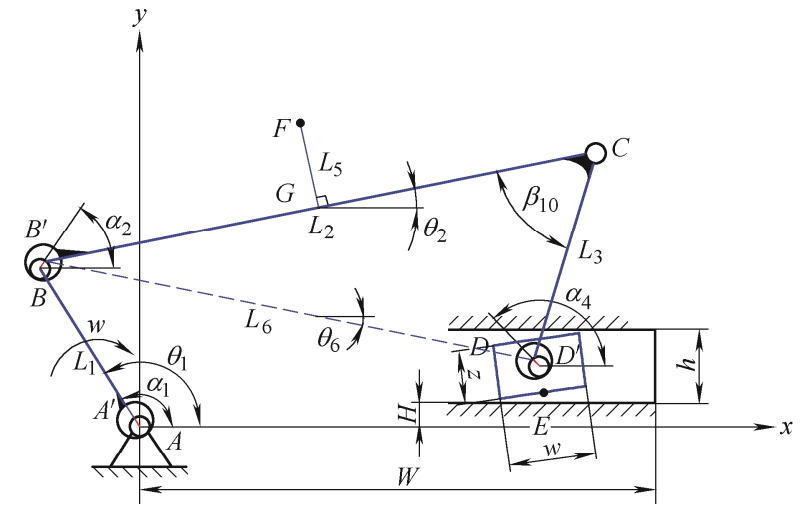

(a) 构态 1 : 含多源不确定性曲柄滑块机构

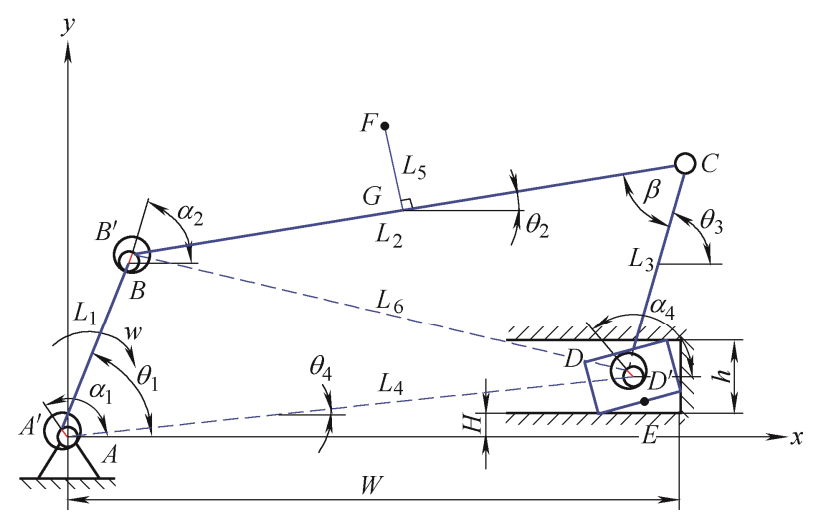

(b) 构态 2: 含多源不确定四连杆机构

图 2 含多源不确定性平面五杆变胞机构位姿模型

\section{1 连杆尺寸不确定性建模}

交变温度工况下, 连杆热变形不容忽视, 杆长 尺寸公差由制造公差和热变形耦合组成。

符合制造精度要求的连杆尺寸分布在一定 范围内, 根据相关标准可得杆件极限偏差, 进而 得到杆件长度上下限 $L_{\text {max }} 、 L_{\text {min }}$ 。连杆制造公差 在工程中常被认为服从正态分布, 标准差可由 $3 \sigma$ 原则求得。连杆尺寸均值 $u_{L}$ 和标准差 $\sigma_{L}$ 可表 示为

$$
\left\{\begin{array}{l}
u_{L}=\left(L_{\max }+L_{\min }\right) / 2 \\
\sigma_{L}=\left(L_{\max }-L_{\min }\right) / 6
\end{array}\right.
$$

由文献[20-21]可知, 连杆尺寸变量应服从截断 正态分布, 杆长尺寸变量在截断区间 $\left[L_{\min }, L_{\max }\right]$ 内 的概率密度函数和累计分布函数为 $f(L)$ 和 $F(L)$, 有

$$
\left\{\begin{array}{l}
f(L)=\frac{v}{\sigma_{L}} \phi\left(\frac{L_{i}-u_{L}}{\sigma_{L}}\right) \\
F(L)=v\left[\Phi\left(\frac{L_{i}-u_{L}}{\sigma_{L}}\right)-\Phi\left(\frac{u_{L}-3 \sigma_{L}}{\sigma_{L}}\right)\right]
\end{array}\right.
$$

式中, $\phi(\cdot)$ 和 $\Phi(\cdot)$ 分别为标准正态分布的概率密度 函数和累计分布函数

$$
v=\left[\Phi\left(\frac{L_{\max }-u_{L}}{\sigma_{L}}\right)-\Phi\left(\frac{L_{\min }-u_{L}}{\sigma_{L}}\right)\right]^{-1}=\frac{1}{2 \Phi(3)-1}
$$

修正后的连杆尺寸均值和方差可表示为

$$
\left\{\begin{array}{l}
\bar{u}_{L}=u_{L} \\
\bar{\sigma}_{L}^{2}=\sigma_{L}^{2}\left\{1-\left[\frac{\phi\left(\frac{L_{\min }-u_{L}}{\sigma_{L}}\right)-\phi\left(\frac{L_{\max }-u_{L}}{\sigma_{L}}\right)}{\Phi\left(\frac{L_{\max }-u_{L}}{\sigma_{L}}\right)-\Phi\left(\frac{L_{\min }-u_{L}}{\sigma_{L}}\right)}\right]^{2}\right. \\
\frac{L_{\max }-u_{L}}{\sigma_{L}} \phi\left(\frac{L_{\max }-u_{L}}{\sigma_{L}}\right)-\frac{L_{\min }-u_{L}}{\sigma_{L}} \phi\left(\frac{L_{\min }-u_{L}}{\sigma_{L}}\right) \\
\frac{\Phi\left(\frac{L_{\max }-u_{L}}{\sigma_{L}}\right)-\Phi\left(\frac{L_{\min }-u_{L}}{\sigma_{L}}\right)}{\sigma_{L}^{2}\left[1-\frac{6 \phi(3)}{2 \Phi(3)-1}\right]=0.973 \sigma_{L}^{2}}
\end{array}\right\}
$$

本文中考虑温度载荷的不确定性时, 可通过区 间变量对其进行数学描述, 如下

$$
T_{\text {min }} \leqslant T \leqslant T_{\text {max }}
$$

式中, $T 、 T_{\text {max }}$ 和 $T_{\text {min }}$ 分别为温度载荷和温度载荷上 下限。

本文假设连杆在温度载荷下只产生线变形。连 杆热变形表示为

$$
\triangle L=\kappa_{1} \cdot L \cdot\left(T-T^{\prime}\right)
$$

式中, $\kappa_{1}$ 为连杆的热膨胀系数, $T^{\prime}$ 为环境参考温度。

经二次修正后连杆尺寸均值为

$$
\tilde{u}_{L}=\bar{u}_{L_{i}}+\Delta L=u_{L_{i}}+\Delta L
$$

因此，服从截断正态分布的尺寸变量可表示为

$$
L_{i} \sim T N\left(u_{L_{i}}+\Delta L, \bar{\sigma}_{L_{i}}{ }^{2}, L_{\text {min }}+\Delta L, L_{\text {max }}+\Delta L\right)
$$

式中, $i$ 为杆件编号 $(i=1,2,3,5), L_{i}$ 为第 $i$ 根连杆 实际尺寸。

原始尺寸变量和截断尺寸变量的概率密度函数 见图 3, 可见耦合连杆制造公差和热变形后, 截断尺 寸变量均值随连杆热变形值变化而变化, 方差减小。

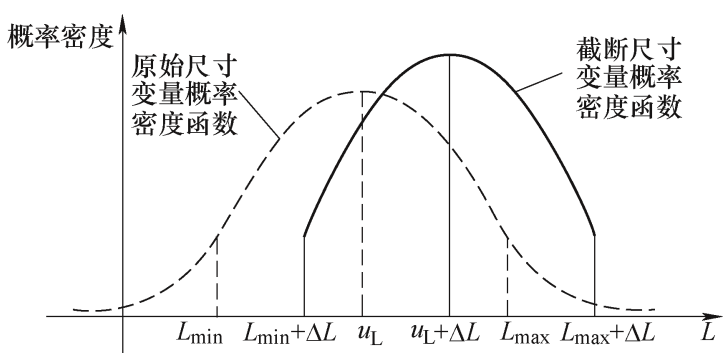

图 3 截断尺寸变量正态分布概率密度函数 


\section{2 转动副间隙不确定性建模}

广义上转动副可简化为轴销-轴套组合 ${ }^{[15]}$, 见 图 4。交变温度工况下, 关节热变形不容忽视 ${ }^{[22]}$, 转动副间隙会由孔轴配合间隙及关节热变形间隙 耦合组成。基于文献[23]可求得各关节孔轴配合 间隙尺寸的均值 $\bar{r}_{k}$ 和标准差 $\sigma_{r_{k}}$, 孔轴配合间隙表 示为

$$
r_{k} \sim N\left(\overline{r_{k}}, \sigma_{r_{k}}{ }^{2}\right)
$$

式中, $k=1,2,4$ 为铰点序号。

根据热变形相关理论, 温度变化时轴销和轴套 线性膨胀，关节热变形间隙可表示为

$$
r_{k}^{\prime}=\kappa_{2} \cdot R_{z t} \cdot\left(T-T^{\prime}\right)-\kappa_{3} \cdot R_{z x} \cdot\left(T-T^{\prime}\right)
$$

式中, $R_{z t} 、 R_{z x}$ 分别为轴套和轴销的半径, $\kappa_{2} 、 \kappa_{3}$ 分 别为轴套与轴销的热膨胀系数。

耦合孔轴配合间隙和热变形间隙后, 转动副间 隙值表示为

$$
\rho_{k}=r_{k}+r_{k}^{\prime}
$$

当机构在运动时轴销在轴套内的位置会出现不 确定性, 因此当机构在运动时将转动副间隙建模为 区间变量更符合工程实际, 比传统的概率建模方法 ${ }^{[24]}$ 具有更高的置信度 ${ }^{[14]}$ 。转动副间隙矢量 $\boldsymbol{c}_{k}$ 可等效为 可变长度的虚拟无质量连杆, 其大小 $c_{k}$ 和方位角 $\alpha_{k}$ 通过区间变量表示为

$$
\left\{\begin{array}{l}
0 \leqslant c_{k} \leqslant \rho_{k} \\
0 \leqslant \alpha_{k} \leqslant 2 \pi
\end{array}\right.
$$

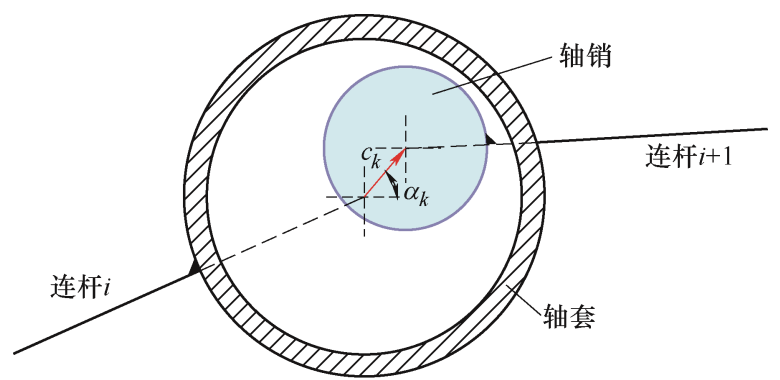

图 4 含间隙转动副模型

\section{3 移动副间隙不确定性建模}

广义上移动副可简化滑块-导轨组合 ${ }^{[25]}$, 由于制 造和装配的不确定性及温度载荷下的热变形, 移动 副不可避免会存在间隙。本文假设滑块、导轨与挡 板为刚性构件, 故不考虑其接触变形。滑块在导轨 中的位姿状态有四种类型，见图 5。

在常温下, 由于制造和装配的不确定性, 移动 副间隙表示为

$$
\chi=(h-z) / 2
$$

本文假设滑块和导轨在温度载荷下只产生线变 形, 故温度载荷下滑块和导轨的热变形可表示为

$$
\left\{\begin{array}{l}
\Delta z=\kappa_{4} \cdot z \cdot\left(T-T^{\prime}\right) \\
\Delta w=\kappa_{4} \cdot w \cdot\left(T-T^{\prime}\right) \\
\Delta h=\kappa_{5} \cdot h \cdot\left(T-T^{\prime}\right)
\end{array}\right.
$$

式中, $\kappa_{4}$ 和 $\kappa_{5}$ 为滑块和导轨的热膨胀系数。

耦合制造和装配的不确定性以及温度载荷下的 热变形，移动副间隙可表示为

$$
\chi^{\prime}=\chi+\Delta h-\Delta z
$$

在温度载荷下, 滑块和导轨的尺寸可表示为

$$
\left\{\begin{array}{l}
z^{\prime}=z+\Delta z \\
w^{\prime}=w+\Delta w \\
h^{\prime}=h+\Delta h
\end{array}\right.
$$

滑块在导轨的位置具有不确定性, 可通过两个 参数来表征滑块位姿不确定性, 即法线方向 ( $y$ 轴) 位置偏差 $\delta p$ 和旋转角度偏差 $\delta \tau$ 。通过区间变量对位 置偏差和旋转角度偏差进行数学描述, 如下

$$
\begin{gathered}
\left\{\begin{array}{l}
-\chi^{\prime} \leqslant \delta p \leqslant \chi^{\prime} \\
-\tau_{\max } \leqslant \delta \tau \leqslant \tau_{\max }
\end{array}\right. \\
\tau=\tau(\delta p)=\arcsin \left(\frac{h^{\prime}-2|\delta p|}{\sqrt{w^{2}+z^{2}}}\right)-\arctan \left(\frac{z}{w}\right)
\end{gathered}
$$

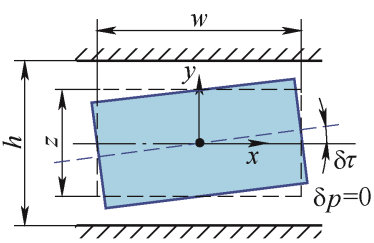

(a) 滑块与导轨无接触

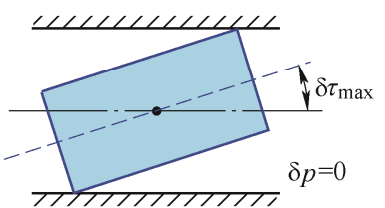

(c) 滑块对角与导轨接触

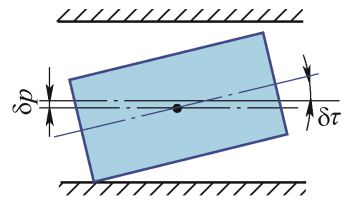

(b) 滑块一角与导轨接触

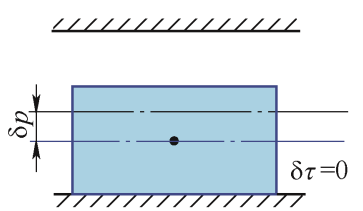

(d) 滑块一侧与导轨全接触
图 5 含间隙移动副模型

\section{3 变胞机构全构态运动可靠性模型}

耦合连杆公差、关节间隙、和热变形等多源不 确定性后, 平面五杆变胞机构输出点 $F$ 的坐标会偏 离理想情况, 输出点 $F$ 的实际坐标表示为 


$$
\begin{aligned}
\left(\begin{array}{l}
F_{x a}^{j} \\
F_{y a}^{j}
\end{array}\right)= & L_{1}\left(\begin{array}{c}
\cos \theta_{1} \\
\sin \theta_{1}
\end{array}\right)+\frac{L_{2}}{2}\left(\begin{array}{c}
\cos \theta_{2} \\
\sin \theta_{2}
\end{array}\right)+c_{1}\left(\begin{array}{c}
\cos \alpha_{1} \\
\sin \alpha_{1}
\end{array}\right)+ \\
& c_{2}\left(\begin{array}{l}
\cos \alpha_{2} \\
\sin \alpha_{2}
\end{array}\right)+L_{5}\left(\begin{array}{l}
\cos \left(\theta_{2}+\pi / 2\right) \\
\sin \left(\theta_{2}+\pi / 2\right)
\end{array}\right)
\end{aligned}
$$

式中, $j$ 为轨迹点号 $(j=1,2, \cdots, n), n$ 为轨迹点 总数。

$$
\left\{\begin{array}{l}
L_{6}=\sqrt{L_{2}^{2}+L_{3}^{2}-2 L_{2} L_{3} \cos \beta_{10}} \\
\theta_{6}=\arcsin \left(\left(L_{1} \sin \theta_{1}+c_{1} \sin \alpha_{1}+\mathrm{c}_{2} \sin \alpha_{2}-H-h / 2-\delta p-c_{4} \sin \alpha_{4}\right) / L_{6}\right)
\end{array}\right.
$$

当滑块与挡板接触时, 变胞机构由构态 1 切换 为构态 2 , 此时滑块与挡板的接触状态有两种类型 (图 6)。

为保证构态切换的连续性, 滑块位置偏差 $\delta p^{\prime}$ 和旋转角度偏差 $\delta \tau^{\prime}$ 在构态切换前后时刻保 持不变, 从而构态 1 滑块的位姿偏差会传递到构 态 2 , 其位姿偏差 $\delta p^{\prime}$ 和 $\delta \tau^{\prime}$ 根据式(17)计算得到。

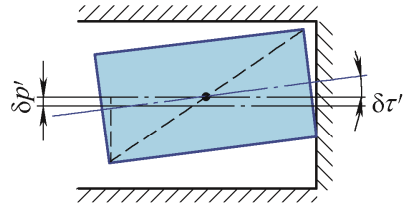

(a) 滑块一角与挡板接触

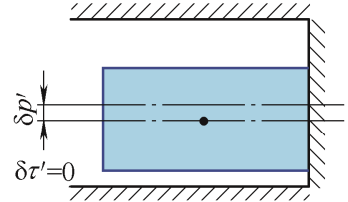

(b) 滑块一侧与挡板接触

图 6 滑块与挡板的接触状态模型

变胞机构子构态 2 为计及多源不确定性四连杆 机构, 参数如下

$$
\left\{\begin{aligned}
\theta_{2}= & 2 \arctan \left(\frac{B \pm \sqrt{A^{2}+B^{2}-C^{2}}}{A+C}\right) \\
A= & L_{1} \cos \theta_{1}-L_{4} \cos \theta_{4}+c_{1} \cos \alpha_{4}+c_{2} \cos \alpha_{3}- \\
& c_{4} \cos \alpha_{4} \\
B= & L_{1} \sin \theta_{1}-L_{4} \sin \theta_{4}+c_{1} \sin \alpha_{1}+c_{2} \sin \alpha_{2}- \\
& c_{4} \sin \alpha_{4} \\
C= & \left(L_{3}{ }^{2}-L_{2}{ }^{2}-A^{2}-B^{2}\right) / 2 L_{2}
\end{aligned}\right.
$$

式中， $L_{4} 、 \theta_{4}$ 为图 $2 \mathrm{~b}$ 中虚拟杆 $A D^{\prime}$ 的杆长和方位 角, 有

$$
L_{6}=\sqrt{\left(L_{1} \sin \theta_{1}+c_{1} \sin \alpha_{1}+c_{2} \sin \alpha_{2}-H-h / 2-\delta p^{\prime}\right)^{2}+\left(\eta-L_{1} \cos \theta_{1}-c_{1} \cos \alpha_{1}-c_{4} \cos \alpha_{4}-z \cdot \sin \delta \tau^{\prime} / 2\right)^{2}}
$$

变胞机构全构态误差传递模型可表示为

$$
\begin{aligned}
g(\boldsymbol{L}, \boldsymbol{I}, T) & =\varepsilon^{A}= \\
& \sqrt{\left(F_{x a}^{j}-F_{x d}^{j}\right)^{2}+\left(F_{y a}^{j}-F_{y d}^{j}\right)^{2}}
\end{aligned}
$$

式中, $\boldsymbol{L}=\left(L_{1}, L_{2}, L_{3}, L_{5}\right)$ 表示随机参数变量组成的

$$
\left\{\begin{array}{l}
L_{4}=\sqrt{\left(W-\frac{w \cos \delta \tau 0^{\prime}}{2}-\frac{z \sin \delta \tau^{\prime}}{2}\right)^{2}+\left(H+\frac{h}{2}+\delta p^{\prime}\right)^{2}} \\
\theta_{4}=\arctan \left(\frac{H+\frac{h}{2}+\delta p^{\prime}}{W-\frac{w \cos \delta \tau^{\prime}}{2}-\frac{z \sin \delta \tau^{\prime}}{2}}\right)
\end{array}\right.
$$

含多源不确定性的平面五杆变胞机构, 构态切 换条件表示如下。

（1）变胞机构在子构态 1 时, 变胞关节 $C$ 锁定 且滑块解锁, 此时其夹角 $\beta$ 为定值 $\left(\beta=\beta_{10}\right)$; 当滑 块上点 $E$ 的横坐标 $E_{x d} \geqslant \eta$ 时, 变胞机构从子构态 1 切换为子构态 $2, E_{x d}$ 和 $\eta$ 的值计算如式(24)。

（2）变胞机构在子构态 2 时, 变胞关节 $C$ 解锁 且滑块锁定, 滑块上点 $E$ 的横坐标为定值, 有 $E_{x d}=\eta$, 变胞关节 $C$ 的夹角 $\beta$ 为变量。当 $\beta>\beta_{10}$ 时, 变胞机构从子构态 2 切换为子构态 $1, \beta$ 的值计算 如式(25)

$$
\left\{\begin{array}{c}
E_{x d}=L_{1} \cos \theta_{1}+c_{1} \cos \alpha_{1}+c_{4} \cos \alpha_{4}+\frac{z}{2} \sin \delta \tau+ \\
\sqrt{L_{6}{ }^{2}-\left(\begin{array}{l}
\left.L_{1} \sin \theta_{1}+c_{1} \sin \alpha_{1}+c_{2} \sin \alpha_{2}\right)^{2} \\
-H-h / 2-\delta p
\end{array}\right)^{2}} \\
\eta=W-\frac{w}{2} \cos \delta \tau^{\prime} \\
\beta=\arccos \left(L_{2}^{2}+L_{3}^{2}-L_{6}{ }^{2} / 2 L_{2} L_{3}\right)
\end{array}\right.
$$

式中， $L_{6}$ 为图 $2 \mathrm{~b}$ 中虚拟杆 $B^{\prime} D$ 的杆长，有

矢量, $\boldsymbol{I}=\left(c_{k}, \alpha_{k}, \delta_{p}, \tau\right)$ 表示区间参数变量组成的矢 量, $\left(F_{x d}^{j}, F_{y d}^{j}\right)$ 为理想情况下机构输出点 $F$ 的坐标。

机构运动可靠度定义为各轨迹点处的实际运动 误差落在允许误差范围内的综合概率。假设各轨迹 
点对应的主动杆转角为 $\theta_{1}^{j}$, 给定允许位置误差限 $\varepsilon$ 后, 机构运动至各轨迹点处的可靠度为

$$
R\left(\theta_{1}^{j}\right)=\operatorname{Pr}\left\{\varepsilon_{\text {max }}^{A} \leqslant \varepsilon, \forall \theta_{1}^{j} \in\left[\theta_{1}^{1}, \theta_{1}^{n}\right]\right\}
$$

式中, $\theta_{1}^{1}$ 表示曲柄初始角度, $\theta_{1}^{n}$ 表示曲柄终止 角度。

由随机和区间变量混合引起的机构运动误差也 是一个区间, 可通过区间优化算法求得运动误差上 限，优化模型可表示为

$$
\begin{array}{ll}
\max & \varepsilon^{A} \\
\text { s.t. } & 0 \leqslant c_{k} \leqslant \rho_{k} \\
& 0 \leqslant \alpha_{k} \leqslant 2 \pi \\
& -\chi \leqslant \delta p \leqslant \chi \\
& -\tau_{\max } \leqslant \delta \tau \leqslant \tau_{\max }
\end{array}
$$

基于蒙特卡洛算法(MCS)求解机构运动可靠 性, 求解流程见图 7。

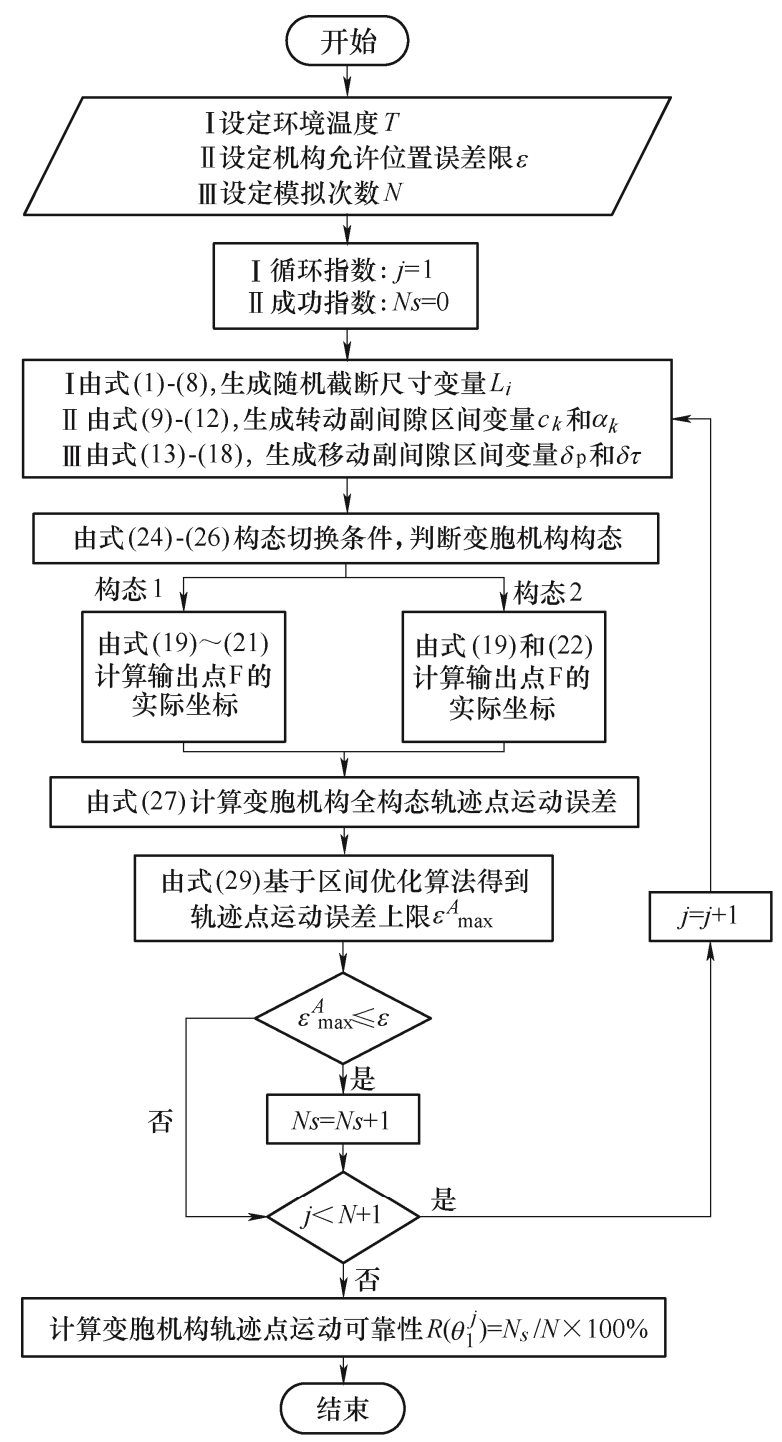

图 7 平面变胞机构运动可靠性计算流程
机构输出轨迹点失效概率 $P_{f}\left(\theta_{1}^{j}\right)$ 为

$$
P_{f}\left(\theta_{1}^{j}\right)=1-R\left(\theta_{1}^{j}\right)
$$

\section{4 案例分析及讨论}

以含多源不确定性的平面五杆变胞机构为例 (图 2), 验证本文所提方法的可行性与实用性。在交 变温度工况下，对变胞机构的转动副间隙、移动副 间隙、连杆公差、连杆和关节的热变形等不确定性 影响因素加以考虑, 以变胞机构一个工作周期内轨 迹点失效概率作为性能评价指标, 运用本文所提方 法分析变胞机构全构态运动可靠性。仿真次数 $N$ 设 为 $10^{5}$, 使样本量足够大, 以确保较高的模拟精度。 驱动杆 $A B$ 初始角度设为 $160^{\circ}$, 顺时针旋转, 在一 个工作周期内等分取 18 个轨迹点进行运动可靠性 分析。变胞机构设计参数的取值见表 1 , 工作环境 温度名义值设为 $-80 \sim 120{ }^{\circ} \mathrm{C}$, 常温设为 $20{ }^{\circ} \mathrm{C}$, 同 时对温度引入 $\pm 2{ }^{\circ} \mathrm{C}$ 的不确定性偏差。

表 1 机构设计参数的名义值

\begin{tabular}{lc}
\hline \multicolumn{1}{c}{ 参数 } & 数值 \\
\hline 杆 $A B$ 名义尺寸 $L_{1} / \mathrm{mm}$ & 150 \\
杆 $B C$ 名义尺寸 $L_{2} / \mathrm{mm}$ & 500 \\
杆 $C D$ 名义尺寸 $L_{3} / \mathrm{mm}$ & 250 \\
杆 $F G$ 名义尺寸 $L_{5} / \mathrm{mm}$ & 150 \\
较点 $A$ 到挡板距离 $W / \mathrm{mm}$ & 540 \\
偏心距 $H / \mathrm{mm}$ & 50 \\
滑块长 $w / \mathrm{mm}$ & 80 \\
滑块高 $Z / \mathrm{mm}$ & 50 \\
变胞关节初始夹角 $\beta_{10} /\left({ }^{\circ}\right)$ & 75 \\
关节轴销和轴套名义直径 $/ \mathrm{mm}$ & 15 \\
轴销和滑块的热膨胀系数 $\left(\times 10^{-6} /{ }^{\circ} \mathrm{C}\right)$ & 15 \\
轴套和导轨的热膨胀系数 $\left(\times 10^{-6} /{ }^{\circ} \mathrm{C}\right)$ & 12 \\
\hline
\end{tabular}

\section{1 平面五杆变胞机构运动可靠性分析算例}

在定量分析变胞机构全构态运动可靠性时, 设 仿真参数分别为: 转动关节配合取 $\mathrm{H} 8 / \mathrm{f} 7$, 移动副间 隙取 $0.02 \mathrm{~mm}$ (常温), 连杆制造公差取 IT7, 连杆热 膨胀系数取 $-1 \times 10^{-6} /{ }^{\circ} \mathrm{C}$, 允许位置误差限 $\varepsilon$ 取 $0.08 \mathrm{~mm}$ 。

变胞机构全构态轨迹点在 $-80 \sim 120{ }^{\circ} \mathrm{C}$ 交变温 度环境下运动可靠性分布规律见图 8 。轨迹点失效 概率越大, 表明轨迹点运动可靠性越低。轨迹点 1 4 和 $13 \sim 18$ 为构态 1 , 轨迹点 $5 \sim 12$ 为构态 2 。

从图 8 可看出, 在相同温度环境下, 全构态轨 
迹点最大失效概率出现在构态 1 ; 随温度变化, 变 大小不同, 这是由关节和连杆的热变形不确定性所 胞机构全构态轨迹点失效概率变化趋势大体一致，导致。

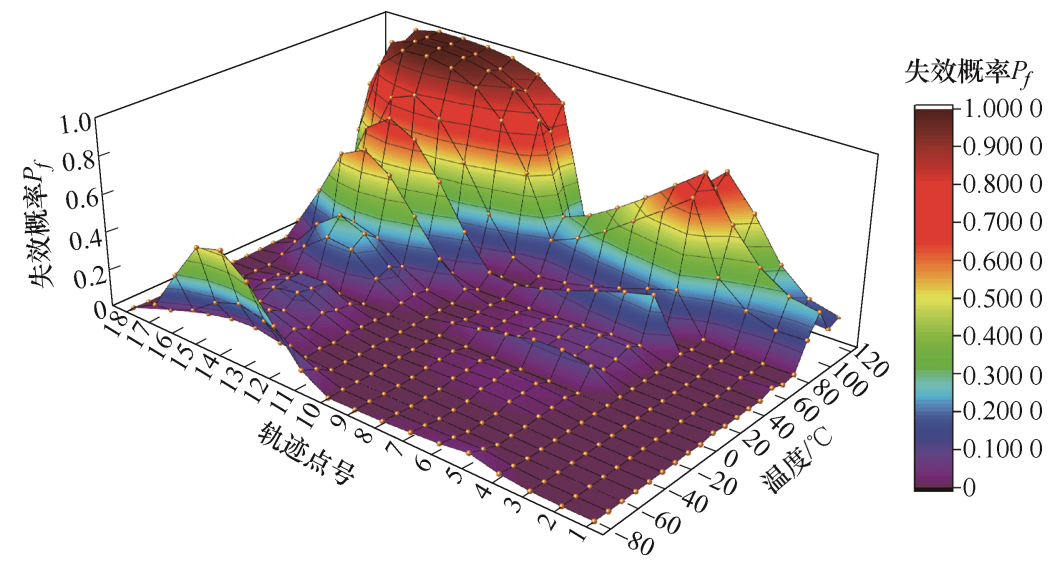

图 8 变胞机构轨迹点运动可靠性

随温度载荷的变化, 变胞机构在构态切换时刻 前、后轨迹点失效概率差值见表 2。由表可知, 变 胞机构在构态切换时刻前轨迹点失效概率小于后轨
迹点失效概率, 即变胞机构在构态切换后轨迹点运 动可靠性降低。

表 2 构态切换时刻前后轨迹点失效概率差值

\begin{tabular}{ccc||ccc||ccc}
\hline 温度 $/{ }^{\circ} \mathrm{C}$ & 差值 $($ 构态 $1 \rightarrow 2)$ & 差值 $($ 构态 $2 \rightarrow 1)$ & 温度 $/{ }^{\circ} \mathrm{C}$ & 差值 $($ 构态 $1 \rightarrow 2)$ & 差值 $($ 构态 $2 \rightarrow 1)$ & 温度 $/{ }^{\circ} \mathrm{C}$ & 差值 $($ 构态 $1 \rightarrow 2)$ & 差值 $($ 构态 $2 \rightarrow 1)$ \\
\hline-80 & -0.0338 & -0.1731 & -10 & -0.0194 & -0.0328 & 60 & -0.257 & -0.229 \\
-70 & -0.0157 & -0.0735 & 0 & -0.0001 & -0.0002 & 70 & -0.0007 & -0.0199 \\
-60 & -0.0001 & -0.0204 & 10 & -0.099 & -0.0788 & 80 & -0.2091 & -0.2412 \\
-50 & -0.0014 & -0.0454 & 20 & -0.0394 & -0.0984 & 90 & -0.3645 & -0.1343 \\
-40 & -0.0021 & -0.0224 & 30 & -0.0525 & -0.076 & 100 & -0.2219 & -0.0573 \\
-30 & -0.0106 & -0.0502 & 40 & -0.0784 & -0.1831 & 110 & -0.2588 & -0.0787 \\
-20 & -0.0118 & -0.0358 & 50 & -0.0431 & -0.1164 & 120 & -0.1741 & -0.0482 \\
\hline
\end{tabular}

本文以单构态轨迹点最大失效概率表征子构态 的运动可靠性, 交变温度下两构态轨迹点最大失效 概率见图 9。在相同的不确定性参数下, 变胞机构
两构态轨迹点最大失效概率随温度变化而波动, 但 构态 1 的失效概率总大于构态 2 , 即构态 2 的运动 可靠性高于构态 1 。

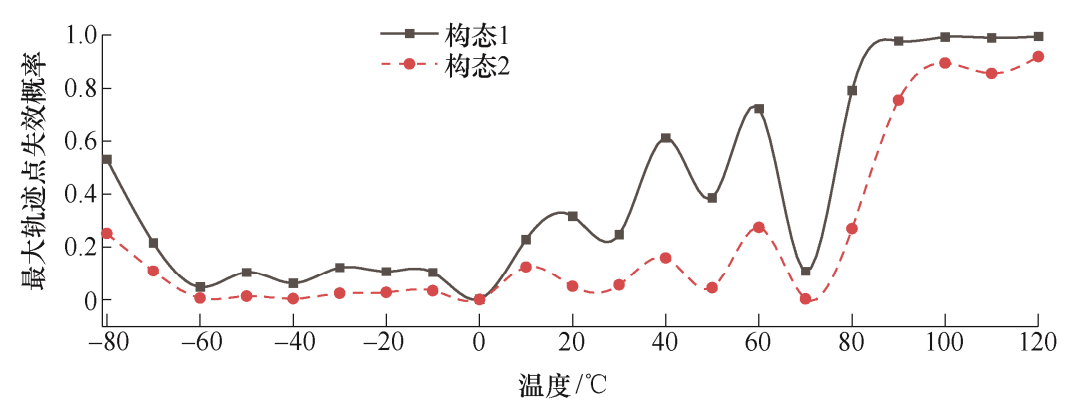

图 9 交变温度下变胞机构两构态运动可靠性

\section{2 运动可靠性影响因素分析算例}

上一节分析了交变温度工况下含多源不确定性 的变胞机构全构态运动可靠性, 需进一步探究单一 不确定性影响因素在交变温度下对变胞机构运动可 靠性的影响规律。
(1) 孔轴配合的影响。以 $\mathrm{H} 7 / \mathrm{h} 7 、 \mathrm{H} 8 / \mathrm{f} 7$ 和 H9/d9 三种优先配合为例, 阐述在交变温度下孔轴配合间 隙和热变形间隙的耦合作用机理。变胞机构转动关 节均取相同的配合形式, 基于转动副间隙模型可得 到各关节配合间隙均值和标准差(表 3), 分别代表 
小、中和大三种间隙配合。

表 3 配合间隙均值和标准差

\begin{tabular}{ccc}
\hline 配合类型 & 均值 $/ \mathrm{mm}$ & 标准差 $/ \mathrm{mm}$ \\
\hline $\mathrm{H} 7 / \mathrm{h} 7$ & 0.0072 & 0.0018 \\
$\mathrm{H} / \mathrm{f} 7$ & 0.0192 & 0.0027 \\
$\mathrm{H} 9 / \mathrm{d} 9$ & 0.0465 & 0.0051 \\
\hline
\end{tabular}

图 10 为交变温度下三种配合下转动副间隙值 及关节失效概率图。在交变温度下, 转动副间隙值 由配合间隙和热变形间隙耦合形成。由式(9) (11) 知, 配合间隙服从正态分布, 耦合热变形间隙后,

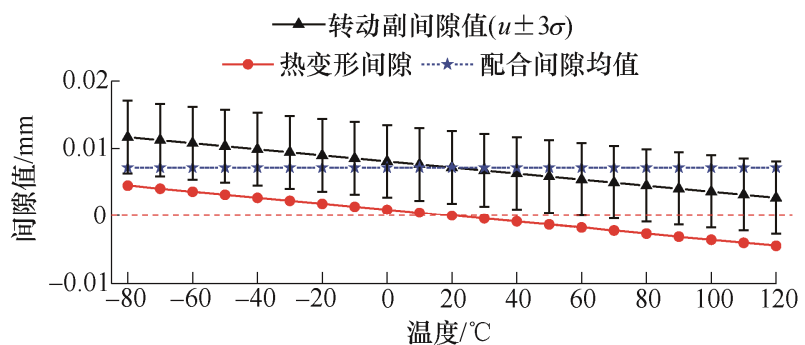

(a) $\mathrm{H} 7 / \mathrm{h} 7$

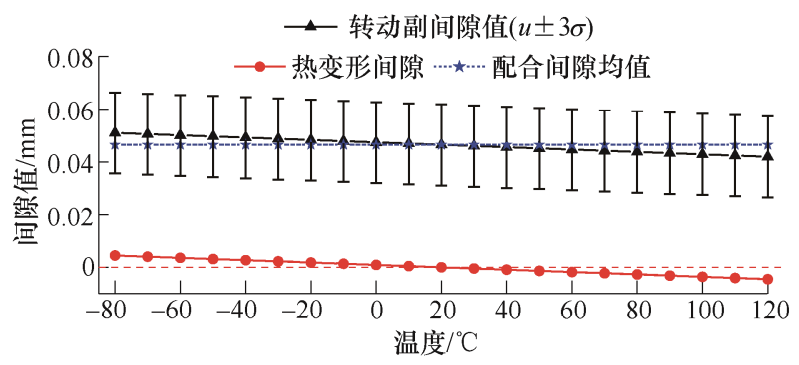

(c) $\mathrm{H} 9 / \mathrm{d} 9$
转动副间隙值亦服从正态分布, 根据 $3 \sigma$ 原则可得转 动副间隙值极限值。

由图 10a、10d 可知, 当配合类型为 $\mathrm{H} 7 / \mathrm{h} 7$ 时, 交变温度在 $-80 \sim 60{ }^{\circ} \mathrm{C}$ 内转动副间隙大于零, 即机 构总能正常运转, 故关节失效概率为零; 交变温度 在 $60 \sim 120{ }^{\circ} \mathrm{C}$ 内转动副间隙会有一定概率会小于 零且关节失效概率随温度升高急剧增大，即关节可 能出现卡死。由图 $10 \mathrm{~b} \sim 10 \mathrm{~d}$ 可知, 当配合类型为 $\mathrm{H} 8 / \mathrm{f} 7$ 和 $\mathrm{H} 9 / \mathrm{d} 9$ 时, 交变温度在 $-80 \sim 120{ }^{\circ} \mathrm{C}$ 内转动 副间隙大于零, 关节失效概率始终为零, 即机构能 正常工作。

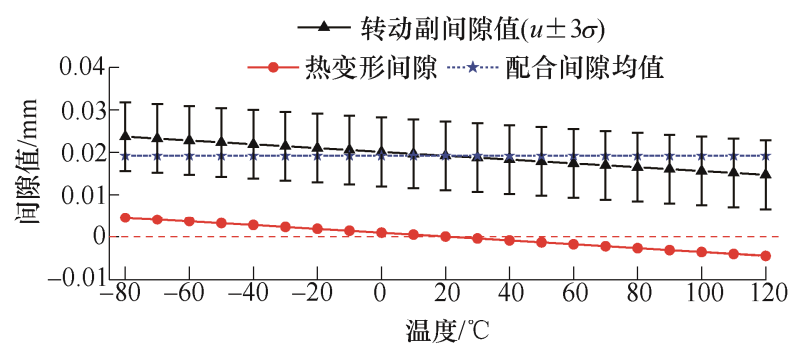

(b) $\mathrm{H} 8 / \mathrm{f7}$

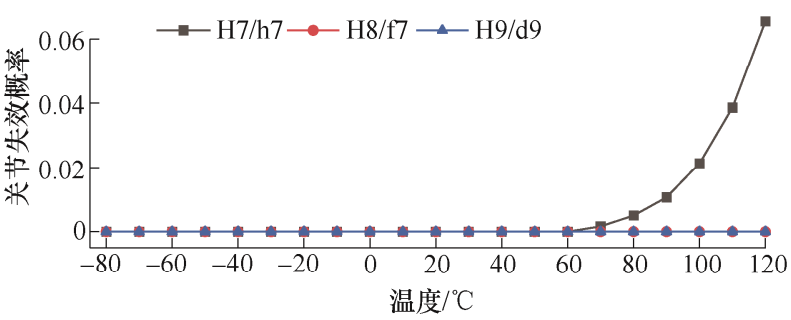

(d) 关节失效概率

图 10 关节间隙值和关节失效概率

以 $\mathrm{H} 8 / \mathrm{f} 7$ 和 $\mathrm{H} 9 / \mathrm{d} 9$ 两种配合为例, 对比分析孔 轴配合对变胞机构全构态运动可靠性的影响规律。 移动副间隙取 $0.02 \mathrm{~mm}$ (常温), 连杆制造公差等级取 IT7, 连杆热膨胀系数取 $-1 \times 10^{-6} /{ }^{\circ} \mathrm{C}$, 机构允许位 置误差限 $\varepsilon$ 设为 $0.1 \mathrm{~mm}$ 。

交变温度下不同配合的两构态轨迹点最大失效
概率见图 11。在相同孔轴配合下，构态 1 的轨迹点 最大失效概率总大于构态 2 ; 轨迹点最大失效概率 随着温度变化而波动; 在 $-80 \sim 120{ }^{\circ} \mathrm{C}$ 内, 大间隙配 合 $(H 9 / d 9)$ 下两构态轨迹点最大失效概率整体大于 中间隙配合 $(\mathrm{H} 8 / \mathrm{f} 7)$; 当温度在 $40 \sim 120{ }^{\circ} \mathrm{C}$ 内, 中间 隙配合下轨迹点最大失效概率急剧上升。

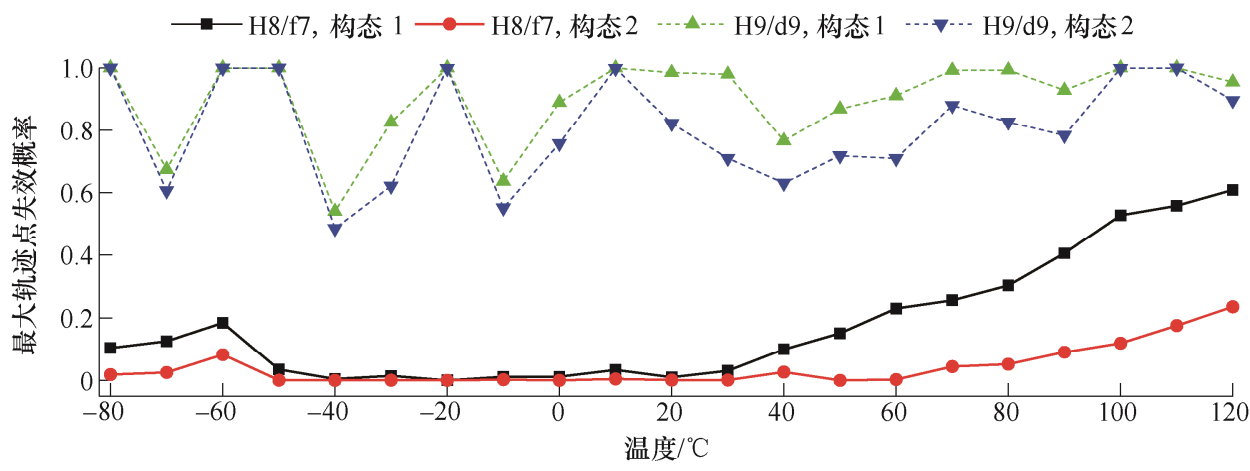

图 11 孔轴配合对机构运动可靠性的影响 
(2) 移动副间隙的影响。常温工况下移动副间 隙不确定性由制造和装配误差引起, 而在交变温度 工况下移动副热变形间隙不容忽视。在交变温度下 移动副热变形间隙值见图 12 。由图可知, 在 $-80{ }^{\circ} \mathrm{C}$ 时移动关节热变形间隙值达到最小-0.015 $1 \mathrm{~mm}$ 。为 保证移动关节能正常运转, 移动副间隙是不可避免 的, 故由制造和装配误差引起的间隙值需大于 $0.0151 \mathrm{~mm}$, 即常温下移动副间隙值需大于 $0.0151 \mathrm{~mm}$ 。

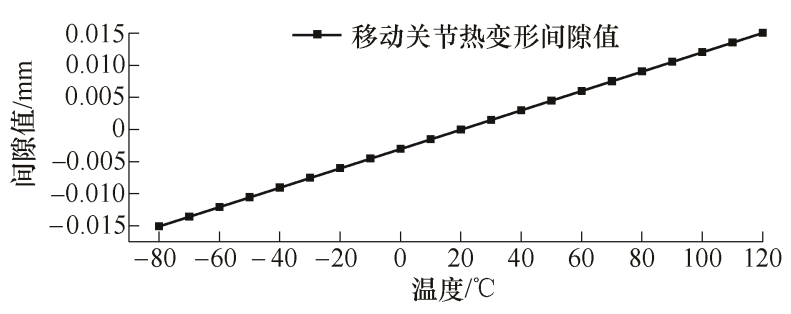

图 12 交变温度下移动关节热变形间隙

移动副间隙对变胞机构构态切换有重要影 响, 有必要进一步将滑块在挡板处的随机接触状 态进行研究。以移动副间隙 $0.02 \mathrm{~mm}$ 为例, 分析 滑块在挡板处的随机接触状态, 见图 13。由图可 知, 滑块的旋转角度偏差区间大小和滑块位置偏 差相关, 且滑块位置偏差绝对值越小, 滑块旋转 角度偏差区间越大。

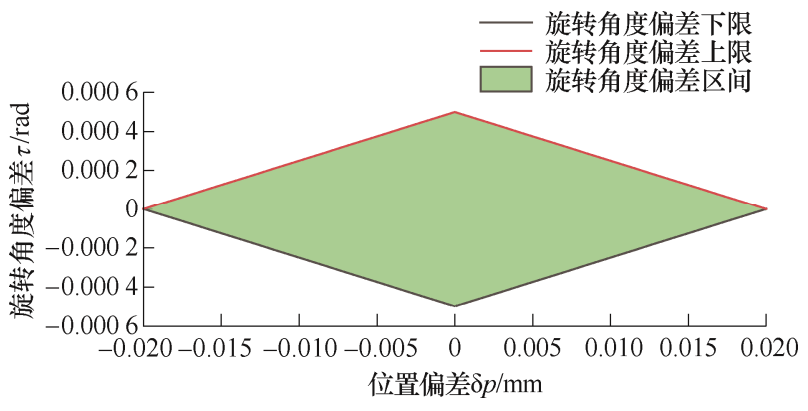

图 13 滑块的位置偏差和旋转角度偏差

为分析移动副间隙值对变胞机构运动可靠性的 影响规律, 转动关节配合取 H8/f7, 连杆公差等级取 IT7, 连杆热膨胀系数取 $-1 \times 10^{-6} /{ }^{\circ} \mathrm{C}$, 移动副间隙取 $0.02 \sim 0.05 \mathrm{~mm}$ (常温下), 机构允许位置误差限 $\varepsilon$ 设 为 $0.12 \mathrm{~mm}$, 进行不同移动副间隙值下变胞机构运 动可靠性对比分析。

交变温度下不同移动副间隙的变胞机构的运动 可靠性见图 14。构态 1 的轨迹点最大失效概率总体 总大于构态 2 ; 温度载荷在 $-80 \sim 20{ }^{\circ} \mathrm{C}$ 低温区间且 移动副间隙在 $0.02 \sim 0.035 \mathrm{~mm}$ 内, 变胞机构两构态 轨迹点最大失效概率较低; 当温度上升和移动副间 隙的增大，变胞机构两构态轨迹点最大失效概率急 剧增大; 在温度载荷在 $120{ }^{\circ} \mathrm{C}$ 且移动副间隙为 $0.05 \mathrm{~mm}$ 的极端工况下构态 1 和 2 的两构态轨迹点 最大失效概率可达 0.9405 和 0.6827 。

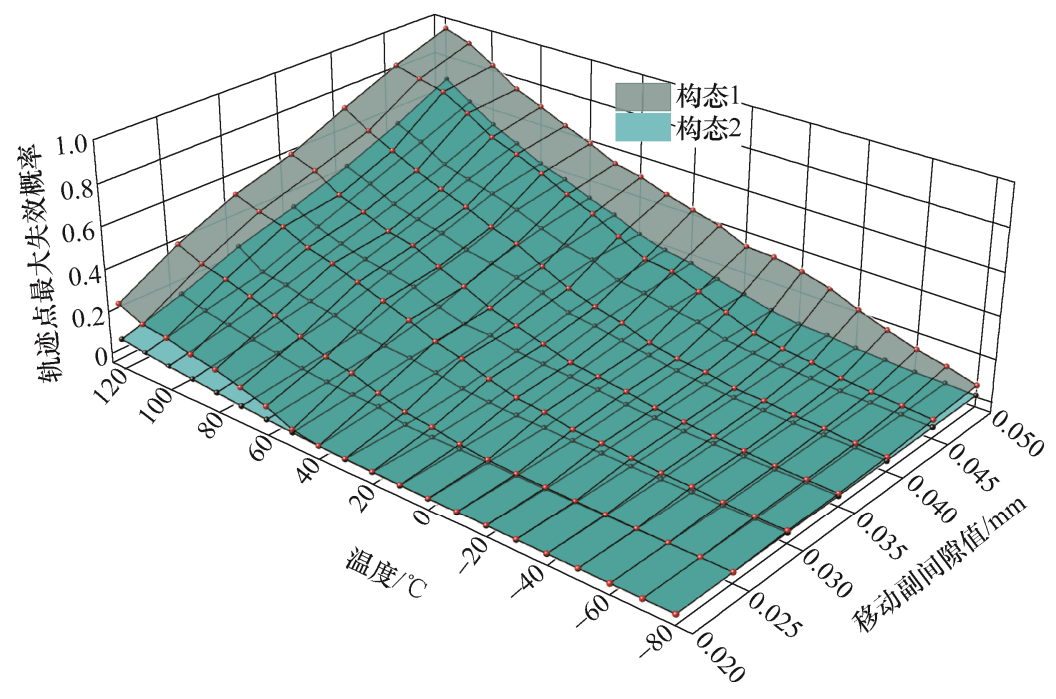

图 14 移动副间隙对机构运动可靠性的影响

（3）连杆尺寸不确定性的影响。交变温度工况 下, 连杆误差不确定性由连杆制造公差和热变形耦 合形成。有必要分析连杆公差等级和热膨胀系数对 变胞机构运动可靠性的影响。

为分析连杆制造公差对变胞机构运动可靠性的 影响, 转动关节配合取 $\mathrm{H} 8 / \mathrm{f} 7$, 移动副间隙取 $0.02 \mathrm{~mm}$ (常温), 连杆热膨胀系数取 $-1 \times 10^{-6} /{ }^{\circ} \mathrm{C}$, 连
杆公差等级取 IT $1 \sim \mathrm{IT} 9$ ，机构允许位置误差限 $\varepsilon$ 设 为 $0.11 \mathrm{~mm}$, 进行不同连杆公差等级下变胞机构运 动可靠性对比分析。

交变温度下不同连杆公差等级的两构态轨迹点 最大失效概率见图 15 。温度载荷在 $-60 \sim 70^{\circ} \mathrm{C}$ 区间 且连杆公差等级在 IT1 IT7 内, 两构态轨迹点最大 失效概率没有明显变化; 当连杆公差等级由 IT7 降 
低到 IT9, 两构态轨迹点最大失效概率急剧上升, 构态 1 和 2 最大失效概率可达 0.5238 和 0.3713 ; 在 $-80 \sim-60{ }^{\circ} \mathrm{C}$ 和 $70 \sim 120{ }^{\circ} \mathrm{C}$ 内, 两构态轨迹点失 效概率较大, 连杆公差等级的变化不能有效降低两 构态轨迹点失效概率。

为分析连杆热膨胀系数对变胞机构运动可靠性 的影响, 转动关节配合取 $\mathrm{H} 8 / \mathrm{f7}$, 移动副间隙取 $0.02 \mathrm{~mm}$ (常温), 连杆公差等级取 IT7, 机构允许位 置误差限 $\varepsilon$ 设为 $0.11 \mathrm{~mm}$, 连杆热膨胀系数 $\kappa_{1}$ 取
$(-3 \sim 3) \times 10^{-6} /{ }^{\circ} \mathrm{C}$, 进行不同连杆热膨胀系数下变胞 机构运动可靠性对比分析

交变温度下不同连杆热膨胀系数下的两构态轨 迹点最大失效概率见图 16 。构态 1 的轨迹点最大失 效概率总大于构态 2 ; 当温度载荷在 $-40 \sim 40{ }^{\circ} \mathrm{C}$ 内, 变胞机构两构态轨迹点最大失效概率均较小; 当温 度在 $-80 \sim-40{ }^{\circ} \mathrm{C}$ 和 $40 \sim 120{ }^{\circ} \mathrm{C}$ 内, 随热膨胀系数 正向或反向增大, 两构态轨迹点最大失效概率急剧 增大。

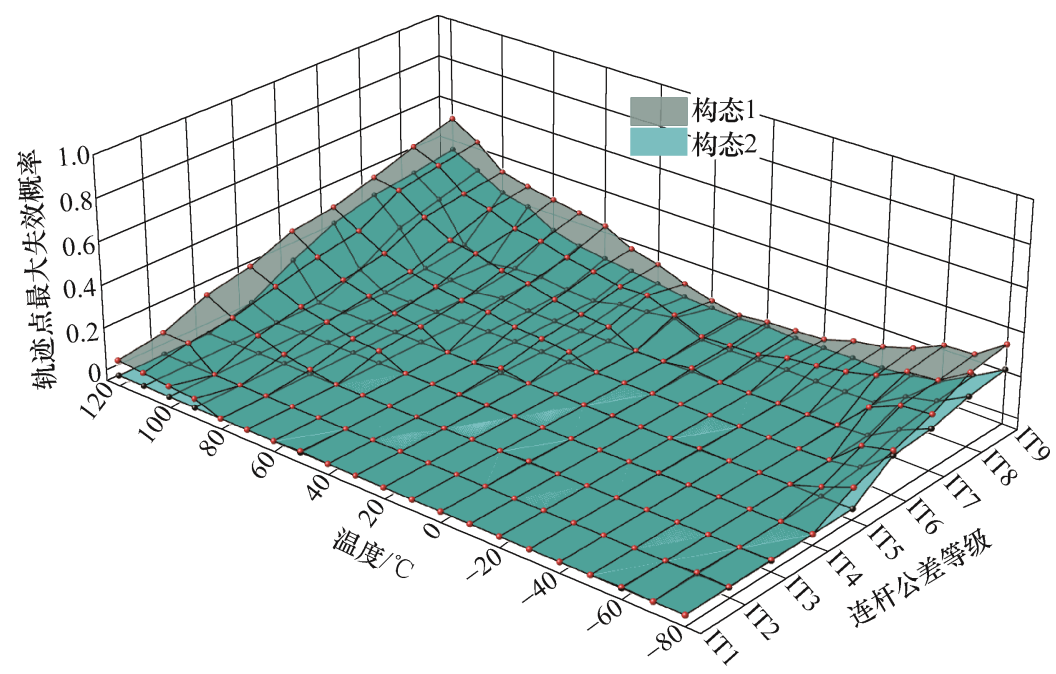

图 15 连杆制造公差对机构运动可靠性的影响

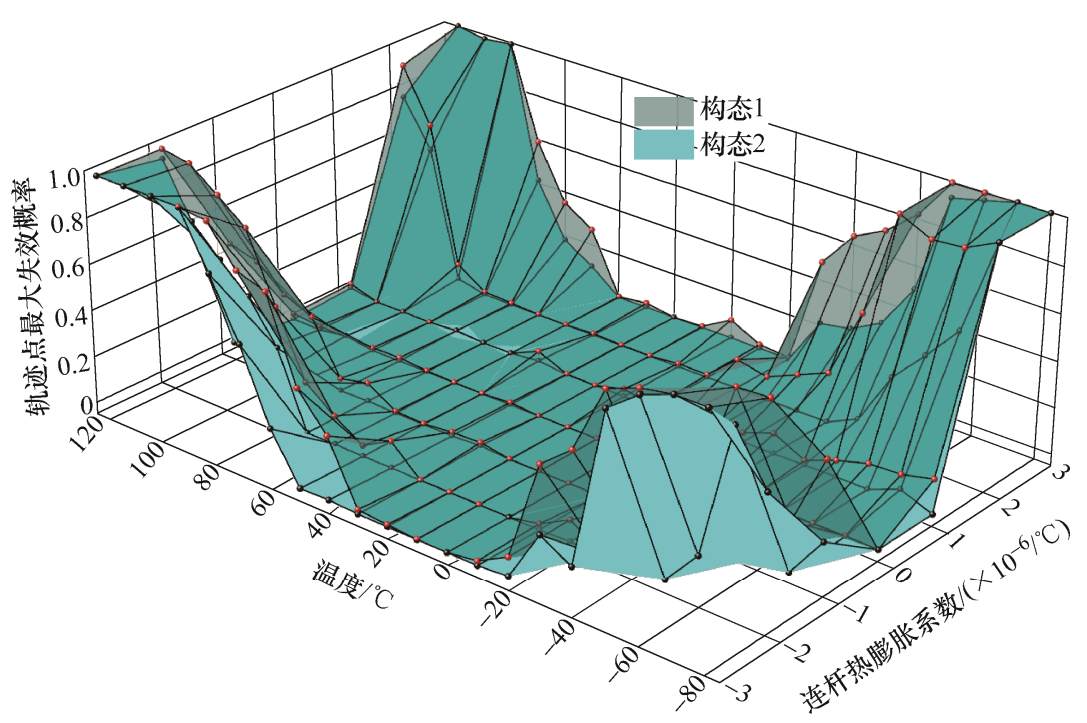

图 16 连杆热膨胀系数对机构运动可靠性的影响

综上所述，变胞机构在同等不确定参数下，构态 1 的运动可靠性总低于构态 2 ; 在交变温度工况下, 需 要根据关节热变形情况合理选择配合, 保证机构的正 常运行, 结合变胞机构两构态运动可靠性选出最佳孔 轴配合; 在 $-80 \sim 20^{\circ} \mathrm{C}$ 内移动副间隙在 $0.02 \sim 0.035 \mathrm{~mm}$ 内, 变胞机构两构态轨迹点最大失效概率均较低; 在
$-60 \sim 70^{\circ} \mathrm{C}$ 内连杆公差等级在 IT1 IT7 之间, 变胞机 构两构态轨迹点最大失效概率均较低, 可适当选取低 的公差等级; 当温度载荷在 $-40 \sim 40^{\circ} \mathrm{C}$ 内, 变胞机构两 构态轨迹点最大失效概率随连杆热膨胀系数变化较 小，而当温度在 $-80 \sim-40^{\circ} \mathrm{C}$ 和 $40 \sim 120^{\circ} \mathrm{C}$ 内，两构态 轨迹点最大失效概率急剧增大。 


\section{5 结论}

（1）考虑关节间隙、连杆制造公差、连杆和关 节热变形等多源不确定性, 基于不确定性建模方法, 将连杆尺寸建模为服从截断正态分布的随机参数, 将转动副和移动副间隙建模为区间参数, 完成对不 确定性影响因素的数学建模。

（2）根据变胞机构可变拓扑结构特性, 建立平 面五杆变胞机构全构态误差传递模型; 结合区间优 化算法和 MCS 方法, 提出一种适应交变温度工况 的含多源不确定性变胞机构全构态运动可靠性分析 方法。

（3）针对交变温度工况，设计阶段需要根据关 节热变形情况合理选择配合, 保证机构的正常运行, 进一步结合变胞机构两构态运动可靠性选出最佳孔 轴配合; 在 $-80 \sim 20{ }^{\circ} \mathrm{C}$ 内移动副间隙在 $0.02 \sim$ $0.035 \mathrm{~mm}$ 内, 变胞机构轨迹点可靠性较高; 在-60 $70{ }^{\circ} \mathrm{C}$ 内连杆公差等级在 IT $1 \sim \mathrm{IT} 7$ 之间, 变胞机构 两构态轨迹点可靠性均较高, 可适当选取精度较低 的公差等级, 降低工艺成本; 当温度载荷在 -40 $40{ }^{\circ} \mathrm{C}$ 内, 变胞机构两构态轨迹点可靠性随连杆热膨 胀系数变化较小, 而在 $-80 \sim-40{ }^{\circ} \mathrm{C}$ 和 $20 \sim 120{ }^{\circ} \mathrm{C}$ 内, 两构态轨迹点可靠性对温度变化极其敏感。

本研究能有效分析交变温度工况下计及多源不 确定性的变胞机构全构态运动可靠性, 对指导变胞 机构的可靠性设计具有理论指导意义。在同等不确 定性参数下，变胞机构在不同的构态表现出不同运 动可靠性, 可进一步建立全构态运动可靠性优化模 型, 实现变胞机构全构态可靠性综合评估。

\section{参 考 文 献}

[1] 王德伦, 戴建生. 变胞机构及其综合的理论基础 [J]. 机 械工程学报, 2007, 43(8): 32-42.

WANG Delun, DAI Jiansheng. Theoretical foundation of metamorphic mechanism and its synthesis[J]. Journal of Mechanical Engineering, 2007，43(8): 32-42.

[2] 李端玲, 张忠海, 戴建生, 等. 变胞机构的研究综述与 展望[J]. 机械工程学报, 2010, 46(13): 14-21.

LI Duanling, ZHANG Zhonghai, DAI Jiansheng, et al. Overview and prospects of metamorphic mechanism[J]. Journal of Mechanical Engineering, 2010, 46(13): 14-21.

[3] SUN D, CHEN G. Kinematic accuracy analysis of planar mechanisms with clearance involving random and epistemic uncertainty $[\mathrm{J}]$. European Journal of
Mechanics-A/Solids, 2016, 58: 256-261.

[4] WANG W, GAO H, ZHOU C, et al. Reliability analysis of motion mechanism under three types of hybrid uncertainties[J]. Mechanism and Machine Theory, 2018, 121: 769-784.

[5] XIAO M, ZHANG J, GAO L, et al. An efficient Kriging-based subset simulation method for hybrid reliability analysis under random and interval variables with small failure probability[J]. Structural and Multidisciplinary Optimization, 2019, 59(6): 2077-2092.

[6] 吴艳荣, 金国光, 李东福. 变胞机构的构态分析及其运 动学仿真研究 $[\mathrm{J}]$. 机械科学与技术, 2006, 25(9): 1092-1095.

WU Yanrong, JIN Guoguang, LI Dongfu. Research on Configuration analysis and kinematic simulation of metamorphic mechanisms[J]. Mechanical Science and Technology, 2006, 25(9): 1092-1095.

[7] ZHANG W, WU T, DING X. An optimization method for metamorphic mechanisms based on multidisciplinary design optimization[J]. Chinese Journal of Aeronautics, 2014, 27(6): 1612-1618.

[8] ZHANG Z, SUN J, WANG Z, et al. Advances in reconfigurable mechanisms and robots II[M]. Springer, 2016.

[9] HU S, ZHANG M, ZHANG B, et al. Design and accuracy analysis of a metamorphic CNC flame cutting machine for ship manufacturing[J]. Chinese Journal of Mechanical Engineering, 2016, 29(5): 930-943.

[10］张满慧, 胡逢源, 胡胜海, 等. 空间变胞机构运动及误 差的全构态四元数模型 $[\mathrm{J}]$. 哈尔滨工程大学学报, 2015, 36(9): 1252-1258.

ZHANG Manhui, HU Fengyuan, HU Shenghai, et al. Kinematics and error modeling based on configuration-complete quaternion for spatial metamorphic mechanisms[J]. Journal of Harbin Engineering University, 2015, 36(9): 1252-1258.

[11] 张义民, 黄贤振, 贺向东. 不完全概率信息牛头刨床机 构运动精度可靠性稳健设计 [J]. 机械工程学报, 2009, 45(4): $105-110$.

ZHANG Yimin, HUANG Xianzhen, HE Xiangdong. Reliability-based robust design for kinematic accuracy of the shaper mechanism under incomplete probability information[J]. Journal of Mechanical Engineering, 2009, 45(4): $105-110$.

[12] 陈放, 李欣玲, 余霞, 等. 平面轨迹机构时变可靠性分 
析的联合概率方法[J]. 机械工程学报, 2017, 53(15):

119-124.

CHEN Fang, LI Xinling, SHE Xia, et al. Joint probability method to time-dependent reliability for planar path mechanisms[J]. Journal of Mechanical Engineering, 2017, 53(15): 119-124.

[13] PANDEY M D, ZHANG X. System reliability analysis of the robotic manipulator with random joint clearances[J]. Mechanism and Machine Theory, 2012, 58: 137-152.

[14] ZHAN Z, ZHANG X, JIAN Z, et al. Error modelling and motion reliability analysis of a planar parallel manipulator with multiple uncertainties[J]. Mechanism and Machine Theory, 2018, 124: 55-72.

[15] ZHAN Z, ZHANG X, ZHANG H, et al. Unified motion reliability analysis and comparison study of planar parallel manipulators with interval joint clearance variables[J]. Mechanism and Machine Theory, 2019, 138: 58-75.

[16] ZHANG J, DU X. Time-dependent reliability analysis for function generator mechanisms[J]. Journal of Mechanical Design, 2011, 133(3): 031005.

[17] ZHANG J, DU X. Time-dependent reliability analysis for function generation mechanisms with random joint clearances[J]. Mechanism and Machine Theory, 2015, 92: 184-199.

[18] WANG Z, WANG Z, YU S, et al. Time-dependent mechanism reliability analysis based on envelope function and vine-copula function[J]. Mechanism and Machine Theory, 2019, 134: 667-684.

[19] GENG X, WANG X, WANG L, et al. Non-probabilistic time-dependent kinematic reliability assessment for function generation mechanisms with joint clearances[J]. Mechanism and Machine Theory, 2016， 104: 202-221.
[20] DU X, HU Z. First order reliability method with truncated random variables[J]. Journal of Mechanical Design, 2012, 134(9): 091005.

[21] BURKARDT J. The truncated normal distribution[J]. Department of Scientific Computing Website, , 2014(1): $1-35$.

[22] 宁峰平, 姚建涛, 安静涛, 等. 适应交变温度的空间轴 承运行可靠性区域分析[J]. 机械工程学报, 2015(15): 103-109.

NING Fengping, YAO Jiantao, AN Jingtao, et al. Analysis of operational reliability region for space bearing in alternating temperature[J]. Journal of Mechanical Engineering, 2015(15): 103-109.

[23] 王兴东, 杨柏, 孔建益, 等. 计及尺寸公差和配合的平 面连杆机构优化设计 [J]. 华中科技大学学报, 2017, 45(3): 23-28.

WANG Xingdong, YANG Bo, KONG Jianyi, e al. Optimal design of planar linkage mechanism with tolerances of dimensional and fit[J]. Journal of Huazhong University of Science and Technology, 2017, 45(3): 23-28.

[24] ZHU J, TING K L. Uncertainty analysis of planar and spatial robots with joint clearances[J]. Mechanism and Machine Theory, 2000, 35(9): 1239-1256.

[25] TING K, HSU K, YU Z, et al. Clearance-induced output position uncertainty of planar linkages with revolute and prismatic joints[J]. Mechanism and Machine Theory, 2017, 111: 66-75.

作者简介: 刘胜利, 男, 1995 年出生, 博士研究生。主要研究方向为机 构学与机器人学。

E-mail: 2437389188@qq.com

唐伟(通信作者), 男, 1986 年出生, 博士, 讲师。主要研究方向为机构 学与机器人学。

E-mail: tangwei@wust.edu.cn 